\title{
IMPACT monitoring network: A better mousetrap
}

$\mathrm{E}$ VERY INFECTIOUS DISEASES OR PUBLIC HEALTH SPECIALIST knows that active disease surveillance is preferable to voluntary reporting because active systems offer more complete and uniform information. The challenge is to find a way to conduct active surveillance that combines a broad catchment with efficient case-finding to minimize labour costs. Such properties are embodied in an innovative new program of the Canadian Pediatric Society and Laboratory Centre for Disease Control (LCDC) called IMPACT (Immunization Monitoring Program, ACTive).

IMPACT is funded by the Childhood Immunization Division of LCDC and one corporate sponsor. Surveillance activities centre upon severe vaccine-associated adverse events, vaccination failures and infections soon to be vaccine-preventable.

The surveillance network is based upon 10 pediatric referral centres extending from St John's to Vancouver. These centres comprise over 2000 pediatric beds and admit over 85,000 children annually, encompassing about $80 \%$ of pediatric admissions to Canadian academic centres. Investigators are infectious diseases specialists, with one exception, and serve as unpaid volunteers. Each supervises a nurse monitor who is employed for 10 to $20 \mathrm{~h}$ per week depending upon the size of the hospital. Monitors have established a network of contacts within each hospital, including infection control nurses, admitting office staff, ward nurses, medical staff, health records personnel and others who help them to find cases of interest. They daily scan admission lists and review potential cases on the wards. Monitors adhere to predetermined case definitions and report their findings on thoroughly tested forms. A coordinating centre in Vancouver receives the reports and manages data entry and analysis. A project manager in Ottawa oversees financial matters and communications. The investigators meet annually to review progress and make key decisions. The project operates

All material presented in Pediatric Infectious Disease Notes has been reviewed and approved by the chairperson, Canadian Pediatric Society Board and representative members of the Canadian Pediatric Society Committee on Infectious Diseases and Immunization in liaison with LCDC. Operating policies and general organization were fine-tuned during an initial 18month pilot project involving five of the centres and subsequent to an external review.

There are numerous surveillance tasks well-suited to such a network. The group has, for example, been enumerating cases of invasive Haemophilus influenzae type b (Hib) infection and was quickly able to demonstrate a sharp decline in the occurrence of cases following the establishment of Hib vaccination programs for young infants. Of all the existing surveillance systems reporting to LCDC, IMPACT was the only one that recognized a substantial number of vaccine failures (from older products) among recent cases. No failures have been identified in fully vaccinated infants but investigators are poised to recommend appropriate investigations if instances occur, illustrating the 'real time' nature of the system. Comparable surveillance is directed at cases of varicella, pertussis and congenital rubella syndrome.

The corporate sponsor is funding a study of pneumococcal disease in children in anticipation of a pneumococcal conjugate vaccine. Over a two-year period, 1000 isolates from invasive infections will be serotyped by collaborators and correlated with clinical and epidemiological data, providing sound information for the final decisions about vaccine composition and routine programs.

The surveillance of vaccine-associated adverse events has been reassuring: thousands of neurological cases have been screened and few were attributable to prior vaccination. Most cases resulted from already established mechanisms, such as febrile seizures after diphtheria-pertussis-tetanus or measles-mumpsrubella vaccines. No instance of paralysis from wild- or vaccine-type polio was detected even though all cases of acute flaccid paralysis are scrutinized by monitors. The system is well-positioned to detect any acute neurological syndromes associated with newly introduced programs such as those for Hib, hepatitis B and, in the near future, varicella and acellular pertussis vaccines. Cases involving serious adverse events are reported to LCDC and provincial epidemiologists within days of 
being recognized, providing a 'finger on the pulse' of childhood immunization unlike any other.

While IMPACT has specific purposes, a functional multicentre network is an asset in approaching other studies. The willingness of Canadian investigators to work together is a noteworthy fact that is being utilized for the betterment of child health.
IMPACT investigators include R Morris (St John's), S Halperin (Halifax), P Déry (Quebec), E Mills (Montreal), M Lebel (Montreal), N MacDonald (Ottawa), R Gold (Toronto, coordinator), B Law (Winnipeg), T Jadavii (Calgary), D Scheifele (Vancouver, coordinator and principal author), V Marchessault (Ottawa, CPS Liaison) and P Duclos (OHawa, LCDC Liaison) 


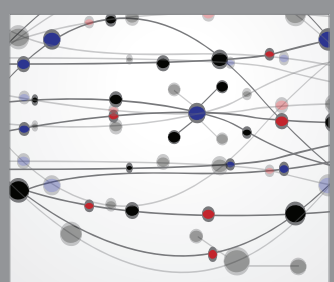

The Scientific World Journal
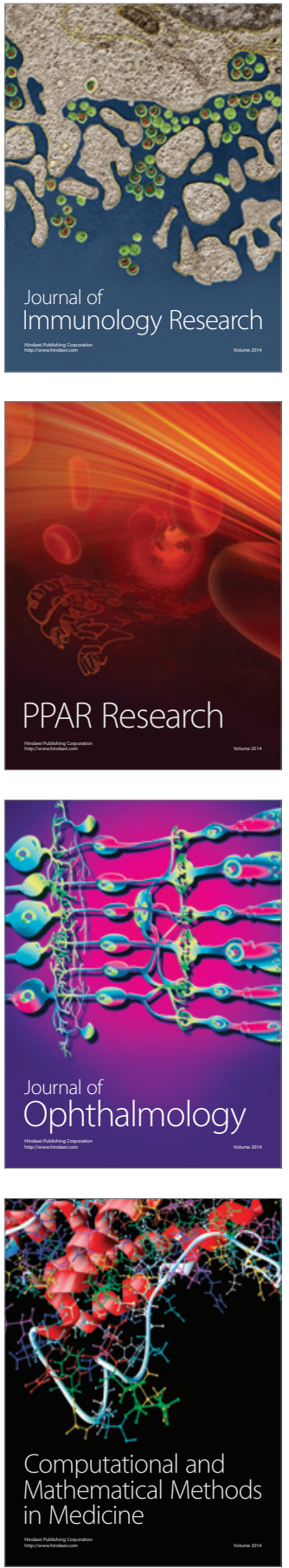

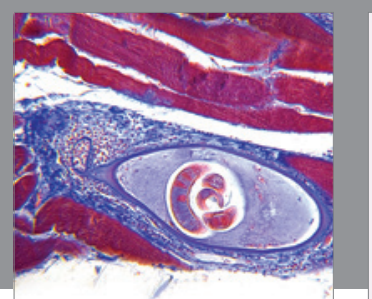

Gastroenterology Research and Practice

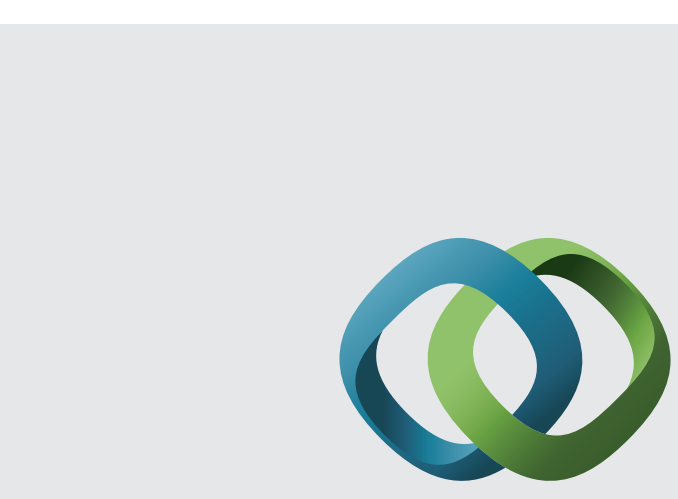

\section{Hindawi}

Submit your manuscripts at

http://www.hindawi.com
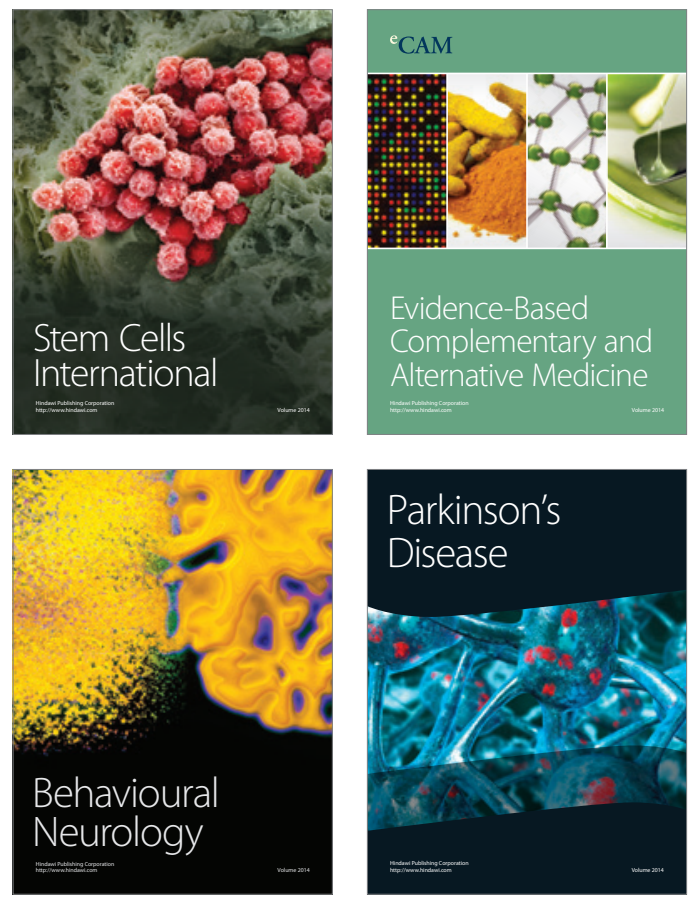
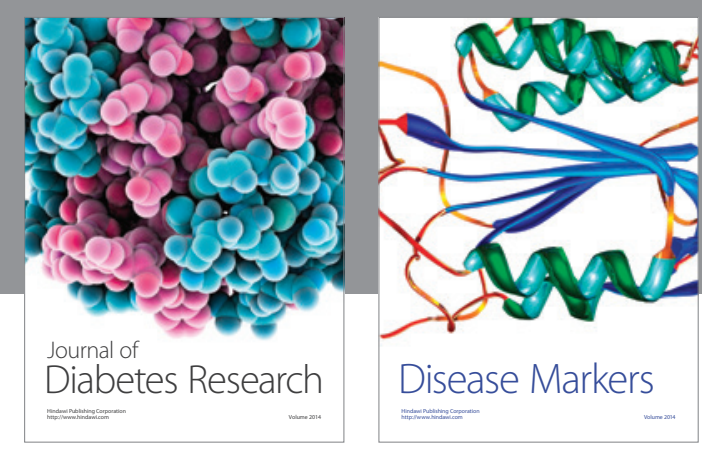

Disease Markers
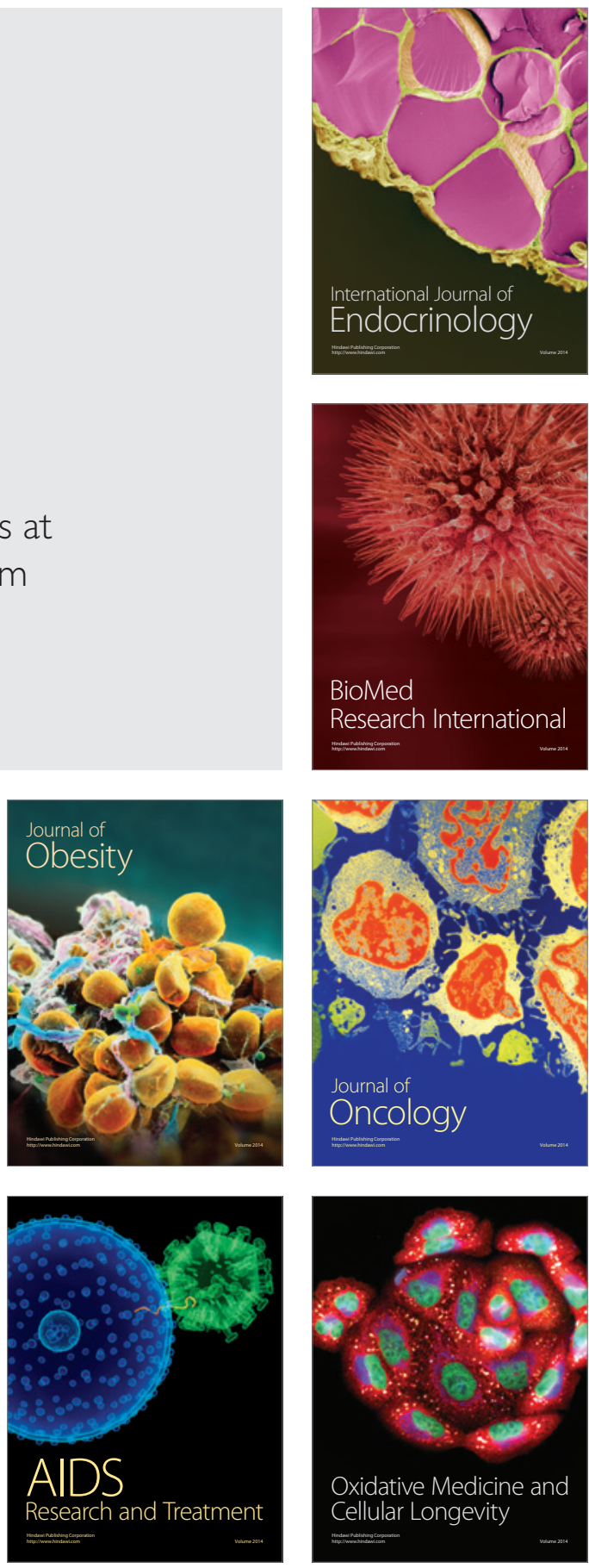